\title{
ANÁlise DA COMPLEXIDADE TEMPORAL DE VARIÁVEIS ClimÁTICAS NO Bioma CaAtinga
}

\section{José Rodrigo Santos Silva ${ }^{1 *}$, Antonio Samuel Alves da Silva ${ }^{2}$, Diego Vicente de Souza Ferreira ${ }^{3}$, Hérica Santos da Silva ${ }^{4}$, Rosilda Benício de Souza ${ }^{3}$, Lázaro de Souto AraúJo ${ }^{5}$}

\author{
${ }^{1}$ Docente do Departamento de Estatística e Ciências Atuariais, Centro de Ciências Exatas e Tecnologia, Universidade Federal de Sergipe \\ ${ }^{2}$ Docente do Departamento de Estatística e Informática, Universidade Federal Rural de Pernambuco. \\ ${ }^{3}$ Docente da Universidade Federal do Cariri \\ ${ }^{4}$ Discente do Doutorado em Biometria e Estatística Aplicada, Universidade Federal Rural de Pernambuco \\ ${ }^{5}$ Docente do Departamento de Ciências Fundamentais e Sociais do Centro de Ciências Agrárias, Universidade Federal da Paraíba \\ *Autor para correspondência: rodrigo.ufs@gmail.com
}

Recebido em 10 de abril de 2016. Aceito em 15 de fevereiro de 2017. Publicado em 30 de junho de 2017.

Resumo - O clima no Brasil é diversificado em consequência de diversos fatores, como a extensão territorial, o relevo e a dinâmica das massas de ar. Em séries temporais, entropia é uma medida da quantidade de incerteza (ou regularidade) com diversas aplicações em séries climáticas. Com o objetivo de compreender a evolução temporal do grau de regularidade em séries temporais climáticas no Bioma Caatinga, e compará-las com o observado por Santos et al. (2015) no bioma Mata Atlântica, aplicamos o método Sample Entropy (SampEn) para analisar a entropia em séries históricas de médias diárias da temperatura do ar, umidade relativa do ar e velocidade do vento, registradas no município de Poço Redondo, no estado de Sergipe, no período de 2005 a 2014. Observamos maiores regularidades para as séries de temperatura do ar (média de 1,12) e umidade relativa do ar (média de 1,28), ou seja, estas variáveis são as que apresentam maior facilidade na realização de previsões climáticas, tal resultado é associado à caracterização climática da região (semiárido). Os valores da entropia observados para a velocidade do vento foram predominantemente maiores que os observados para as demais variáveis (média de 2,18). Não foram observadas tendências nas séries entrópicas, onde todas foram caracterizadas como estacionárias para a janela estudada. Em comparação com os resultados obtidos por Santos et al.(2015), foi possível observar a influência da maritimidade e das massas de ar sobre a entropia da temperatura do ar e umidade relativa do ar.

Palavras-chave: Clima; Sample Entropy; CaAtinga.

\section{Temporal complexity analysis of Climatic variables in the CaAtinga}

Abstract - The climate of Brazil is diversified due to different factors, such as the territorial extension,relief and dynamics of air masses. In time series, entropy is a measure of the quantity of uncertainty (or regularity) with many applications in climate series. The aim of this study was to understand the temporal evolution of the degree of regularity in climate time series in the Caatinga, and compare them with those observed by Santos et al. (2015) in theAtlantic Forest. For this purpose, we applied the Sample Entropy (SampEn) method to analyze the entropy in historical series of daily average of air temperature, air relative humidity, and wind speedrecorded in the municipality of Poço Redondo, in Sergipe State, Brazil, from 2005 to 2014. There was a higher regularity in the air temperature series (mean of 1.12) and air relative humidity (mean of 1.28), i.e., these variables are the easiest onesfor climate predictions. This result is associated with climatic characterization of the semiarid region. The values of entropy observed for wind speed were predominantly higher than those observed for the other 
variables (mean of 2.18). No trends were observed in the entropic series, and all of them were characterized as stationary for the studied window. Compared with the results obtained by Santos et al. (2015), it was possible to observe the influence of the maritimity and air masseson the entropy of the air temperature and air relative humidity.

KeYwords: CLIMATE; SAMPLE ENTROPY; CAATINGA.

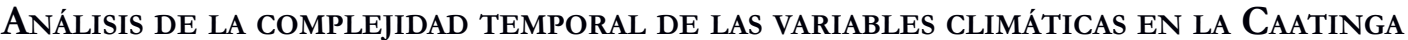

REsumen - El clima de Brasil es diversificado en consecuencia de varios factores, como la extensión territorial, el relieve y la dinámica de las masas de aire. En series temporales, la entropía es una medida de la cantidad de incertidumbre (o regularidad) con muchas aplicaciones en series climáticas. Con el fin de comprender la evolución del grado de regularidad en series temporales climáticas en la Caatinga y compararlas con los resultados de Santos et al. (2015) obtenidos en la Mata Atlántica, se aplicó el método Sample Entropy (SampEn) para analizar la entropía en series históricas de promedios diariosde la temperatura del aire, de la humedad relativa del aire y de la velocidad del viento, registrados en el municipio de Poço Redondo, en el estado de Sergipe, Brasil, en el período de 2005 a 2014. Se observaron regularidades mayores para las series de la temperatura del aire (promedio de 1,12) y de la humedad relativa del aire (promedio de 1,28), indicando que estas variables son las más fáciles de usar para las predicciones del clima. Este resultado está asociado con la caracterización climática de la región (región semiárida).Los valores de entropía observados para la velocidad del viento fueron en su mayoría mayores que los observadas para las otras variables (promedio de 2,18). No se observaron tendencias en las series entrópicas, y todas se caracterizaron como estacionariaspara la ventana estudiada. En comparación con los resultados obtenidos por Santos et al. (2015), fue posible observar la influencia de la maritimidad y de las masas de aire sobre la entropía de la temperatura del aire y de la humedad relativa del aire.

Palabras clave: Clima; Sample Entropy; CaAtinga.

\section{INTRODUÇÃO}

Os recursos naturais são importantes para o desenvolvimento de atividades como a agricultura (Slate et al., 2013), a produção de energia (Pimenta et al., 2008) e a utilização dos recursos hídricos (Li et al., 2014; Li-Hao et al., 2013), etc. Para um melhor planejamento destas atividades é de fundamental importância a compreensão acerca da dinâmica destes recursos. Desta forma, um melhor entendimento da dinâmica do tempo e do clima tonou-se um objetivo de diversos pesquisadores, que buscam entender melhor o comportamento de elementos climáticos (temperatura, umidade do ar, pressão atmosférica, radiação solar, precipitação, nebulosidade, vento). A dificuldade sob tais estudos reside no fato de o tempo e o clima receberem influência de muitos fatores climáticos, como a latitude, a altitude, as massas de ar, a continentalidade, a maritimidade (distância de certo lugar em relação a mares e oceanos), as correntes marítimas, o relevo, a vegetação, o solo, e outros, dificultando a sua modelagem e previsão (Torres et al., 2008).

Dentre todos os elementos climáticos existentes, três deles se destacam pela importância da sua influência sobre o meio, e pelo vasto volume de trabalhos científicos dedicados ao seu estudo. São eles: a temperatura do ar, a umidade relativa do ar e a velocidade do vento. As pesquisas direcionadas ao comportamento da temperatura do ar são importantes para a agricultura, pois indicam os períodos de semeadura, irrigação e colheita, contribuem 
para determinara produtividade agrícola, o zoneamento de risco climático, o crédito e o seguro agrícola (Filho et al., 2008; Ismael-Filho et al., 2015; Pereira et al., 2015). A umidade relativa do ar, que representa a relação entre a quantidade de água existente no ar (umidade absoluta) e a quantidade máxima que poderia haver na mesma temperatura (ponto de saturação), é importante para a meteorologia, pois possibilita saber como o tempo se comportará, sendo a sua estimativa de grande utilidade tanto para o setor agrícola como no planejamento, manejo e gestão dos recursos hídricos (Ismael-Filho et al., 2015; Li et al., 2014; Li-Hao et al., 2013). Já a velocidade do vento apresenta aplicações em estudos sobre a erosão do solo (Jönsson, 1992), a dispersão do pólen e sementes (Jongejans et al., 2001) e a geração de energia (Pimenta et al., 2008), entre outros.

Abordagens que envolvem conceitos das áreas de física, estatística e biologia vêm sendo utilizados para estudar a dinâmica do clima, buscando descrever a variabilidade de séries climáticas no espaço e no tempo (Benicio et al., 2013; Mihailovic et al., 2014; Santos et al., 2015). Um desses conceitos é a entropia, uma grandeza que mede o grau de desordem em um sistema, e que é utilizada como uma medida física-estatística para medir o grau de regularidade em séries temporais, especialmente em séries temporais climáticas. Tais medidas foram utilizadas em estudos sobre os elementos de temperatura do ar (Balzter et al., 2015; Kossobokov et al., 2012; Santos et al., 2015; Shuangcheng et al., 2006; Zhao et al., 2011), da umidade relativa do ar (Li-Hao et al., 2013; Quadro et al., 2012; Santos et al., 2015) e da velocidade do vento (Figueirêdo et al., 2014; Santos et al., 2015). Um dos estimadores da entropia mais utilizados na literatura foi proposta por Shuangcheng et al. (2006), e é denominado de SampleEntropy (SampEn).

O SampEn foi desenvolvido por Richman e Moorman (2000), e utilizado para medir a complexidade de séries temporais climáticas pela primeira vez por Shuangcheng (2006). Eles estudaram a temperatura do ar diária, registradas em estações meteorológicas localizadas nas montanhas do sudoeste da China, obtendo como resultados que as regiões que apresentaram as maiores irregularidades (maior entropia) ocorreram em áreas sob maior influência das massas de ar. Outros estudos na mesma área foram realizados por Kossobokov et al. (2012) na Europa, e por Zhao et al. (2011) na China, entretanto não foram observadas correlações entre as estimativas da entropia com os fatores climáticos. Silva (2014) realizou um estudo com as estimativas da entropia em séries temporais diárias, do período de 1990 a 2012, de temperatura do ar média (máxima, média, mínima e amplitude térmica), umidade relativa do ar média e velocidade média do vento, em 264 estações meteorológicas do Brasil. Seus resultados indicaram que as regularidades das séries climáticas no Brasil foram medianas (valores entre $1 \mathrm{e}$ 2), não sendo observada correlação entre as estimativas de entropia com os fatores climáticos de maritimidade, latitude, vegetação e relevo. Para a temperatura média diária do ar e a temperatura máxima diária do ar, houve indícios de possíveis influências da massa de ar Equatorial Continental sob o fenômeno.

$\mathrm{Na}$ Europa Central, Balzter et al. (2015) verificaram um aumento dos valores da entropia das séries temporais de temperatura média do ar de 1960 a 2014. Eles concluíram que houve um aumento na irregularidade das séries temporais de temperatura média do ar para o período estudado, significando que com o passar do tempo, torna-se mais difícil realizar previsões acerca desta variável. Porém, a pesquisa realizada por Santos et al. (2015) foi observado um comportamento diferente deste, os resultados mostraram uma diminuição de $0,9 \%$ ao ano para a entropia em séries históricas, de 2008 a 2014, da temperatura diária do ar no município de EstânciaSE. Para as outras séries estudadas, a da umidade relativa do ar e a da velocidade do vento, apresentaram um comportamento estacionário em relação a evolução temporal da entropia, sendo que a entropia da temperatura do ar foi predominantemente menor que as outras duas. 
Este trabalho teve por objetivo estudar a evolução temporal da entropia de séries diárias de temperatura do ar, umidade relativa do ar e velocidade do vento, registradas em uma estação meteorológica localizadas no bioma Caatinga, sendo selecionada uma estação localizada no município de Poço Redondo, no estado de Sergipe, Brasil. Desta forma, será possível compararmos os resultados deste trabalho com o obtido por Santos et al. (2015), que utilizou séries históricas registradas em uma estação meteorológica localizada no bioma Mata Atlântica, no litoral deste mesmo estado, Sergipe.

\section{Material e MÉtodos}

\section{Dados}

As observações são séries históricas coletadas a cada três horas (00:00, 03:00, 06:00, 09:00, 12:00, 15:00, 18:00 e 21:00) pela estação agrometeorológica do Instituto Nacional Brasileiro de Pesquisas Espaciais (INPE), e fornecido pelo Centro de Previsão de Tempo e Estudos Climáticos (CPTEC) disponível por meio do endereço

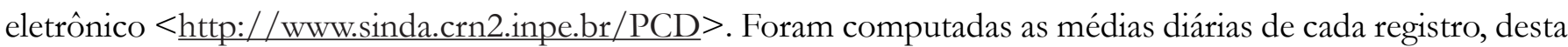
forma as observações representam médias diárias de temperatura do ar $\left({ }^{\circ} \mathrm{C}\right)$, umidade relativa do ar $(\%)$, e velocidade do vento $(\mathrm{m} / \mathrm{s})$. A estação meteorológica fica localizada no município de Poço Redondo, Sergipe, a uma altitude de 160 metros. O município está localizado na mesorregião do Sertão Sergipano, distante a $140 \mathrm{~km}$ da capital Aracaju, dentro do bioma Caatinga (Figura 1).

Figura 1 - Localização do estado de Sergipe no Nordeste brasileiro e a posição georreferenciada das estações agrometeorológicas do INPE nos municípios de Estância (vermelho) e Poço Redondo (azul).

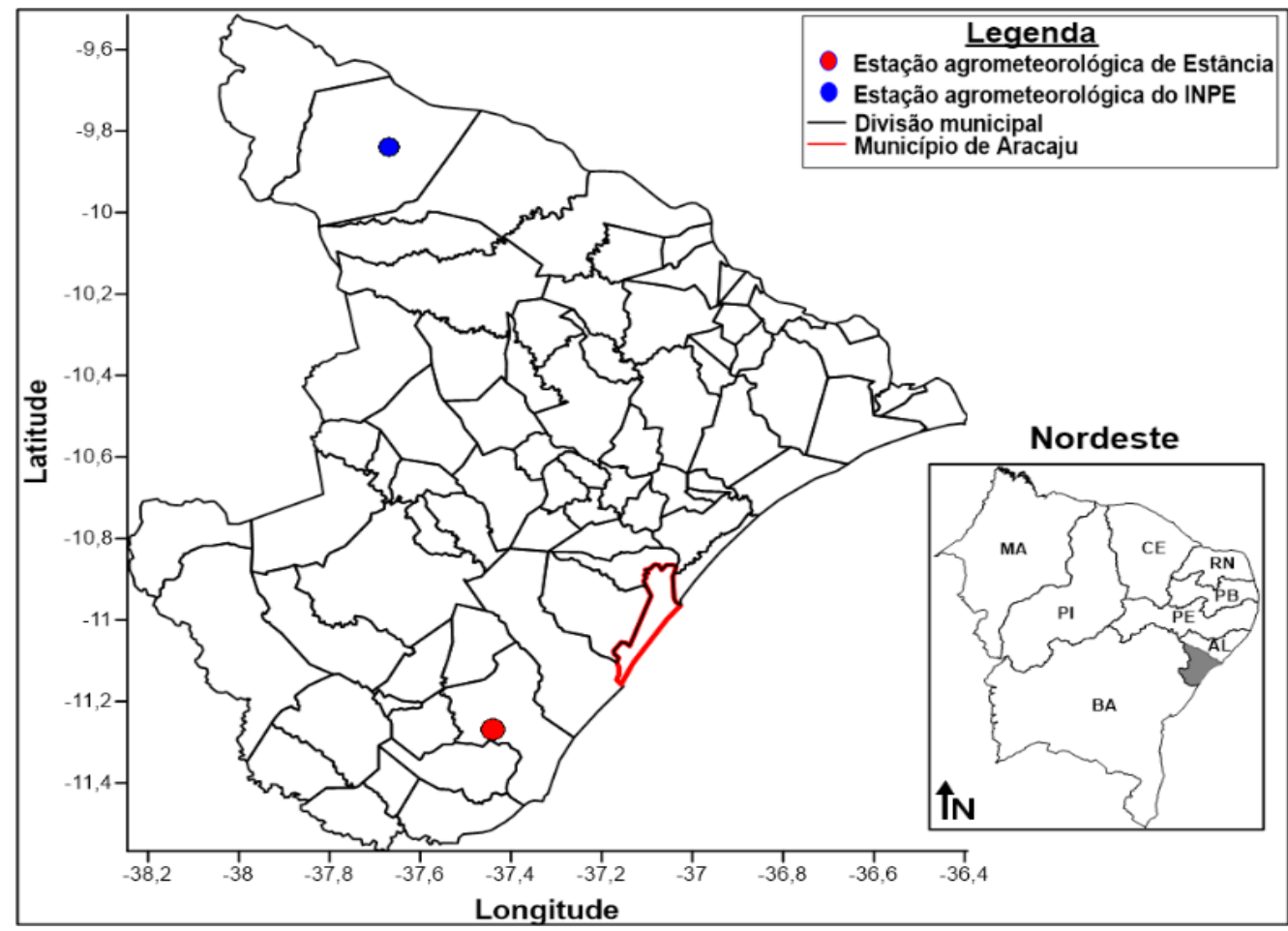

Embora esteja localizado dentro da zona climática Tropical Nordeste Oriental, conforme classificação 
do IBGE (2014), o clima característico da região é o semiárido. Conforme Cruz (2009), o clima em Poço Redondo é caracterizado por baixa umidade e pouco volume pluviométrico, e o período de maior pluviosidade está compreendido entre os meses de abril e julho. Esta característica interfere diretamente na temperatura do ar e na umidade relativa, onde os meses mais chuvosos apresentam menores médias diárias de temperatura do ar e maiores médias diárias da umidade relativa do ar. Estas sazonalidades podem ser vistas de forma clara nas Figuras 2-A e 2-B. Já a velocidade do vento (Figura 2-C) não apresenta nenhum padrão temporal visível. Comparando a localização e os fatores climáticos incidentes sobre as diferentes estações meteorológicas, observamos que o município de Estância também está localizado na zona climática Tropical Nordeste oriental, porém compreendido no bioma Mata Atlântica (IBGE, 2014). Ambas as estações meteorológicas sofrem influência das mesmas massas de ar (Silva, 2014), e possuem um relevo de baixa altitude (IBGE, 2014).

Figura 2 - Médias diárias de: (A) temperatura do ar $\left({ }^{\circ} \mathrm{C}\right),(\mathrm{B})$ umidade relativa do ar $(\%)$ e $(\mathrm{C})$ velocidade do vento $(\mathrm{m} / \mathrm{s})$, no município de Poço Redondo, Sergipe, Brasil. No período de 1 de janeiro de 2005 a 31 de dezembro de 2014.
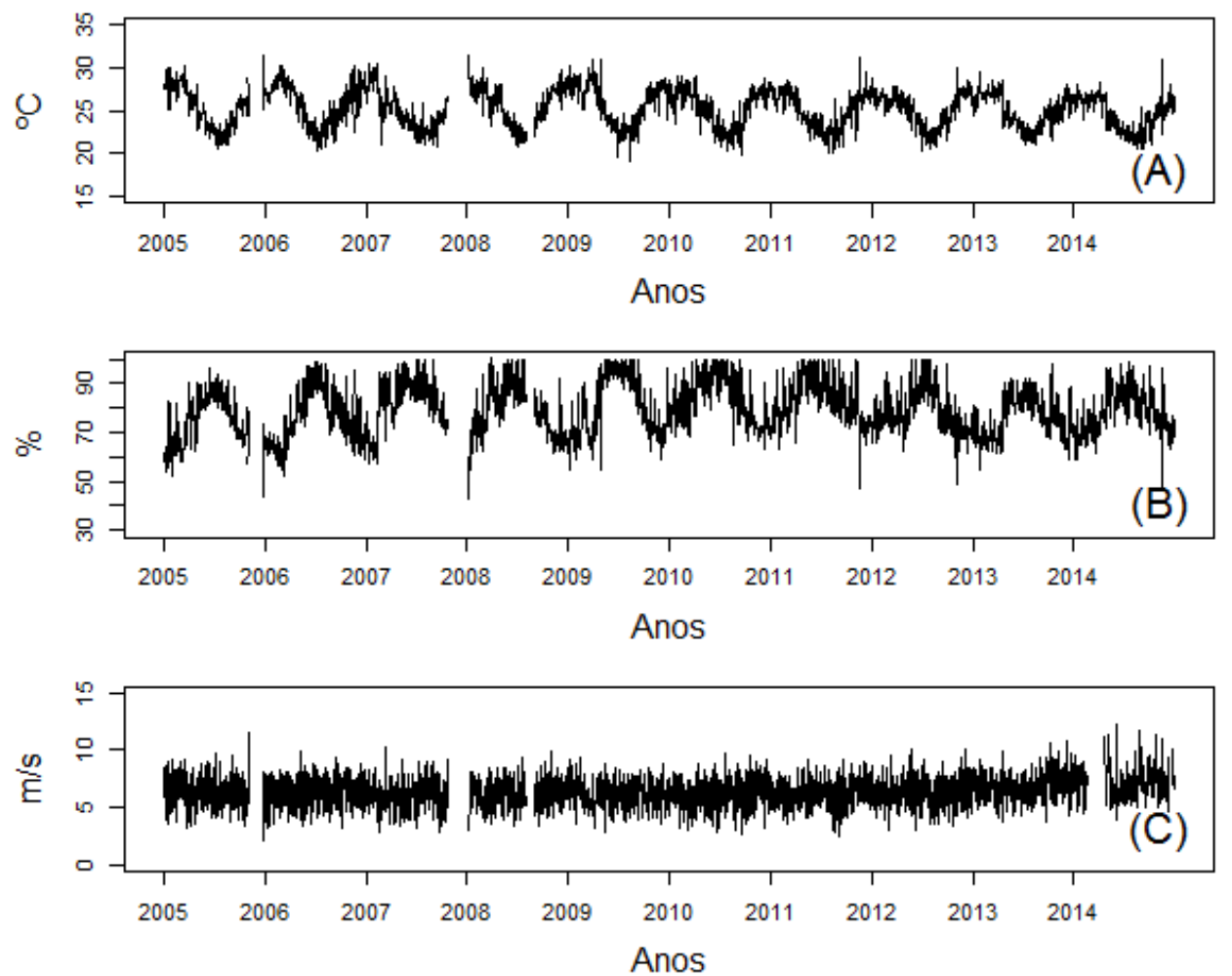

\section{Sample entropy}

O método Sample Entropy, introduzido por Richman e Moorman (2000), é definido como sendo o logaritmo natural da probabilidade condicional de que duas sequências similares (dentro de um nível de tolerância $\mathrm{r})$, para $\mathrm{m}$ pontos, permanecerem similares para $\mathrm{m}+1$ pontos. Seja $\mathrm{x}(1), \ldots, \mathrm{x}(\mathrm{N})$ uma série temporal de tamanho $\mathrm{N}$, o algoritmo de SampEn (m,r,N) é descrito como:

i) Construir vetores de tamanho em que 
$x(i)=[x(i), x(i+1), \ldots, x(i+m-1)], i=1, \ldots, N-m+1$

ii) Definir a distância entre os vetores, onde

$$
d_{m}[x(i), x(j)]=\max _{\mathrm{k}=0, \mathrm{~m}-1}[|x(i+k)-x(j+k)|]
$$

iii) Para cada ; calcula-se

$$
\begin{gathered}
\boldsymbol{B}_{\boldsymbol{i}}^{\boldsymbol{m}}(\boldsymbol{r})=\frac{\boldsymbol{B}_{\boldsymbol{i}}}{(\boldsymbol{N}-\boldsymbol{m}-\mathbf{1})} \\
A_{i}^{m}(r)=\frac{A_{i}}{(N-m)},
\end{gathered}
$$

onde $\mathrm{B}_{\mathrm{i}}$ é o número dos vetores $\mathrm{x}(\mathrm{j})$ de tamanhom que são similares aos vetores $\mathrm{x}(\mathrm{i})$ dentro da distância $\mathrm{r}$ : $d_{m}[x(i), x(j)] \leq r$ e $A_{i}$ é o número dos vetores $x(j)$ de tamanho $(m+1)$ que são similares aos vetores $x(i)$ dentro da distância $\mathrm{r}$;

iv) Calcula-se

$$
\begin{aligned}
& B^{m}(r)=\frac{1}{N-m}\left(\sum_{i=1}^{N-m} B_{i}^{m}(r)\right) \\
& A^{m}(r)=\frac{1}{N-m}\left(\sum_{i=1}^{N-m} A_{i}^{m}(r)\right)
\end{aligned}
$$

onde $\mathrm{B}^{\mathrm{m}}(\mathrm{r})$ é a probabilidade de dois vetores serem similares para $\mathrm{m}$ pontos, $\mathrm{A}^{\mathrm{m}}(\mathrm{r})$ é a probabilidade de dois vetores serem similares para $(m+1)$ pontos;

v) Por fim, calcula-se o índice SampleEntropy:

$$
\operatorname{SampEn}(m, r, N)=-\ln \left(\frac{A^{m}(r)}{B^{m}(r)}\right)
$$

que representa a probabilidade condicional que de duas sequências que são similares para $\mathrm{m}$ pontos permanecem similares para $\mathrm{m}+1$ pontos (quando um dado consecutivo é adicionado).

Sample Entropy é uma modificação do método Approximate Entropy (ApEn) (Pincus et al., 1992). Ambos os métodos servem para quantificar a complexidade em séries temporais não lineares, que foram amplamente usados em análise de processos fisiológicos e séries climáticas (Shuangcheng et al., 2006; Tang et al., 2004). Uma maior complexidade (menor regularidade) da série temporal gera o valor do SampEn mais alto. De acordo com Shuangcheng et al. (2006) os valores da estatística de SampEn estão entre zero (série totalmente regular) e três (série totalmente aleatória).

Para a análise das séries médias diárias das variáveis climáticas adotou-se o valor de r igual a $20 \%$ do desvio 
padrão da amostra e uma janela de observação de 2 dias, ou seja, $\mathrm{r}=0,20 \mathrm{e} \mathrm{m}=2$, conforme sugerido por Richman e Moorman (2000) para o cálculo do SampEn. Todas as estimativas de SampEn para as séries diárias de temperatura do ar, umidade relativa do ar e velocidade do vento $(\mathrm{m} / \mathrm{s})$ foram obtidas por meio de um algoritmo desenvolvido em linguagem $\mathrm{C}$, a partir de modificações implementadas no código fonte disponível no endereço eletrônico (https://www.physionet.org/physiotools/mse/).

\section{RESUlTADOS E DisCUSSÃo}

Um resumo descritivo anual das variáveis em estudo pode ser visto na Tabela 1. Não foi observada nenhuma tendência temporal nas médias anuais da umidade relativa do ar e da temperatura do ar. Como a umidade relativa do ar e o ponto de saturação do vapor são inversamente proporcionais, isso implica que ela também será inversamente proporcional a temperatura do ar (Varejão-Silva, 2006). Desta forma era esperado que o aumento (ou diminuição) da temperatura do ar resulta-se na diminuição (ou aumento) da umidade relativa do ar. Apenas nos anos de 2011 e 2013 , em que a temperatura média anual $\left(24,85^{\circ} \mathrm{C}\right.$ e $24,92^{\circ} \mathrm{C}$, respectivamente) foi menor em comparação aos respectivos anos anteriores $\left(25,30^{\circ} \mathrm{C}\right.$ em 2010 e $25,13^{\circ} \mathrm{C}$ em 2012), a umidade relativa do ar também foi menor em comparação com os respectivos anos anteriores $(83,52 \%, 83,33 \%, 78,1 \%$ e 75,83\%, respectivamente em 2010, 2011, 2012 e 2013). Em relação a velocidade do vento, de 2007 a 2014, nota-se um aumento no valor médio anual, passando de $6,11 \mathrm{~m} / \mathrm{s}$ em 2007 a 7,25 m/s em 2014. Estas médias de velocidade do vento classificam a região como de ventos moderados, conforme a escala de Beaufort (Mendonça et al., 2007). Proporcionalmente as maiores variabilidades são observadas na velocidade do vento, com coeficiente de variação total de 20,16\%, seguida da umidade relativa do ar (coeficiente de variação de 13,00\%) e temperatura do ar (coeficiente de variação de 8,76\%). Esta relação foi observada em todos os anos estudados.

Tabela 1 - Média, desvio padrão (D.P.) e coeficiente de variação (C.V.) das séries históricas anuais de temperatura do ar $\left({ }^{\circ} \mathrm{C}\right)$, umidade relativa do ar $(\%)$ e velocidade do vento $(\mathrm{m} / \mathrm{s})$, no município de Poço Redondo, Sergipe, Brasil. No período de 1 de janeiro de 2005 a 31 de dezembro de 2014.

\begin{tabular}{cccccccccc}
\hline \multirow{2}{*}{ Anos } & \multicolumn{1}{c}{ Temperatura do ar } & \multicolumn{3}{c}{ Umidade Relativa do ar } & \multicolumn{3}{c}{ Velocidade do Vento } \\
\cline { 2 - 10 } & Média & D.P. & C.V. & Média & D.P. & C.V. & Média & D.P. & C.V. \\
\hline $\mathbf{2 0 0 5}$ & 25,27 & 2,43 & 9,62 & 75,40 & 9,93 & 13,17 & 6,31 & 1,37 & 21,71 \\
$\mathbf{2 0 0 6}$ & 25,70 & 2,40 & 9,34 & 75,97 & 10,98 & 14,45 & 6,36 & 1,23 & 19,34 \\
$\mathbf{2 0 0 7}$ & 24,80 & 2,17 & 8,75 & 82,31 & 9,86 & 11,98 & 6,11 & 1,26 & 20,62 \\
$\mathbf{2 0 0 8}$ & 25,83 & 2,22 & 8,59 & 78,17 & 11,17 & 14,29 & 6,12 & 1,23 & 20,10 \\
$\mathbf{2 0 0 9}$ & 25,83 & 2,41 & 9,33 & 80,74 & 12,32 & 15,26 & 6,20 & 1,13 & 18,23 \\
$\mathbf{2 0 1 0}$ & 25,30 & 2,23 & 8,81 & 83,52 & 9,37 & 11,22 & 6,22 & 1,21 & 19,45 \\
$\mathbf{2 0 1 1}$ & 24,85 & 2,07 & 8,33 & 83,33 & 8,90 & 10,68 & 6,25 & 1,17 & 18,72 \\
$\mathbf{2 0 1 2}$ & 25,13 & 1,94 & 7,72 & 78,10 & 8,69 & 11,13 & 6,33 & 1,21 & 19,12 \\
$\mathbf{2 0 1 3}$ & 24,92 & 1,90 & 7,62 & 75,83 & 8,57 & 11,30 & 6,93 & 1,17 & 16,88 \\
$\mathbf{2 0 1 4}$ & 24,54 & 1,86 & 7,58 & 78,13 & 8,24 & 10,55 & 7,25 & 1,52 & 20,97 \\
\hline Série Completa & $\mathbf{2 5 , 2 2}$ & $\mathbf{2 , 2 1}$ & $\mathbf{8 , 7 6}$ & $\mathbf{7 9 , 1 7}$ & $\mathbf{1 0 , 2 9}$ & $\mathbf{1 3 , 0 0}$ & $\mathbf{6 , 4 0}$ & $\mathbf{1 , 2 9}$ & $\mathbf{2 0 , 1 6}$ \\
\hline
\end{tabular}

Ao longo dos 10 anos estudados não foi observado padrões temporais, como tendências, nas entropias estimadas para as 3 variáveis (Tabela 2 e Figura 3). Este resultado é diferente do observado por Balzter et al. 
(2015) e por Santos et al.(2015), onde o primeiro observou um crescimento da entropia para a temperatura do ar, e o segundo, um decrescimento para a mesma variável. Enquanto Balzter et al. (2015) utilizaram uma janela de observação de 54 anos (1960 a 2014), Santos et al.(2015) observou apenas 7 anos (2008 a 2014), sendo este um período suficiente para caracterizar uma tendência de decrescimento da entropia.

Tabela 2. Estimativas anuais do Sample Entropy (SampEn) e os respectivos erros (Std.), em séries temporais de médias diárias de temperatura do ar, umidade relativa do ar e velocidade do vento, no município de Poço Redondo - SE, no período de 2005 a 2015.

\begin{tabular}{ccccccc}
\hline \multirow{2}{*}{ Ano } & \multicolumn{2}{c}{$\begin{array}{c}\text { Temperatura do } \\
\text { Ar }\end{array}$} & \multicolumn{2}{c}{ Umidade Relativa do Ar } & \multicolumn{2}{c}{$\begin{array}{r}\text { Velocidade do Ven- } \\
\text { to }\end{array}$} \\
\cline { 2 - 7 } & SampEn & Std. & SampEn & Std. & SampEm. & Std. \\
\hline $\mathbf{2 0 0 5}$ & 1,065 & 0,037 & 1,142 & 0,046 & 2,075 & 0,025 \\
$\mathbf{2 0 0 6}$ & 1,086 & 0,047 & 1,339 & 0,032 & 2,234 & 0,013 \\
$\mathbf{2 0 0 7}$ & 1,289 & 0,027 & 1,448 & 0,019 & 2,055 & 0,027 \\
$\mathbf{2 0 0 8}$ & 0,994 & 0,055 & 1,005 & 0,060 & 2,125 & 0,016 \\
$\mathbf{2 0 0 9}$ & 1,040 & 0,034 & 0,928 & 0,047 & 2,194 & 0,015 \\
$\mathbf{2 0 1 0}$ & 1,134 & 0,037 & 1,318 & 0,045 & 2,237 & 0,015 \\
$\mathbf{2 0 1 1}$ & 1,244 & 0,032 & 1,453 & 0,023 & 2,268 & 0,013 \\
$\mathbf{2 0 1 2}$ & 1,131 & 0,028 & 1,261 & 0,044 & 2,282 & 0,012 \\
$\mathbf{2 0 1 3}$ & 0,961 & 0,047 & 1,391 & 0,036 & 2,103 & 0,015 \\
$\mathbf{2 0 1 4}$ & 1,227 & 0,031 & 1,560 & 0,024 & 2,191 & 0,015 \\
\hline
\end{tabular}

Figura 3 - Evolução temporal das estimativas anuais do SampEn em séries temporais de médias diárias de temperatura do ar, umidade relativa do ar e velocidade do vento, no município de Poço Redondo - SE, no período de 2005 a 2015.

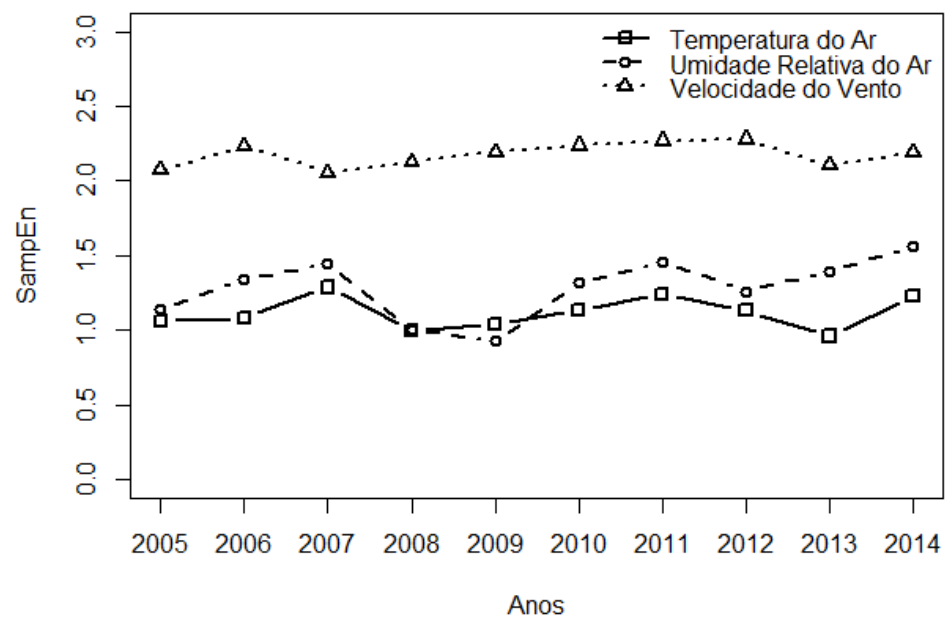

A entropia estimada para a velocidade do vento foi predominantemente maior do que as estimativas para as outras variáveis em ambos os estudos, onde a média da entropia para a temperatura do ar, umidade relativa do ar e velocidade do vento foi 1,12; 1,28 e 2,18; respectivamente, enquanto que Santos et al. (2015) observaram para essas mesmas variáveis uma média da entropia de 1,67; 1,92 e 1,96 respectivamente. Silva (2014) mostrou que a entropia da velocidade do vento não está relacionada com a dinâmica das massas de ar, apesar do fato da maior influência das massas de ar atuantes na região dá-se em áreas litorâneas. Os resultados mostraram que a entropia 
da velocidade do vento estimada (Tabela 2) foi maior ( $\mathrm{p}$-valor $<0,0001)$ do que as observações realizadas por Santos et al. (2015). Em relação à umidade relativa do ar nota-se que a maior regularidade temporal da variável ( $\mathrm{p}$-valor < 0,0001) foi observada em uma região com maior distância de massas de água, no bioma Caatinga. Da mesma forma, foi observado um comportamento semelhante em relação a temperatura do ar, onde as menores entropias ( $\mathrm{p}$-valor $<0,0001)$ foram observadas na região semiárida do estado.

Apenas no ano de 2009 a entropia estimada para a umidade relativa média diária do ar foi menor que a mesma estatística estimada para temperatura média diária do ar (Tabela 2), sendo superior nos demais anos. Dentro da escala proposta por Shuangcheng (2006), em ambas as variáveis as estimativas da entropia foram consideradas moderadas. Segundo Silva(2014), as maiores entropias da temperatura do ar no Brasil são encontradas em regiões de interação de diferentes massas de ar. O estado de Sergipe sofre atuação da massa de ar Tropical Atlântica no inverno, e Equatorial Atlântica no verão, de acordo com IBGE(2002), porém, em ambos os casos as massas de ar citadas acabam atuando com mais força no litoral da região Nordeste, perdendo força antes de atingir o semiárido sergipano. Desta forma, as massas de ar podem ter colaborado para que tenha sido observado uma menor entropia para a temperatura do ar neste estudo. Ou seja, trata-se de estações meteorológicas próximas, e sob influência das mesmas massas de ar, porém com diferentes intensidades.

A principal diferença deste estudo para o realizado por Santos et al. (2015) está na diferente localização das estações meteorológicas. Enquanto neste trabalho a estação meteorológica utilizada para realizar as observações está localizada no bioma Caatinga, no sertão do estado de Sergipe, a estação meteorológica utilizada por Santos et al. (2015) está situada no bioma Mata Atlântica, no litoral deste mesmo estado. Devido à esta localização geográfica de ambas estações meteorológicas, podemos descartar a influência do relevo sob a diferença nos resultados observados neste trabalho, em comparação com Santos et al. (2015). Além disso, de acordo com Silva (2014), a entropia de variáveis climáticas não sofre influência da latitude, onde apenas a temperatura do ar apresentou correlação com a influência de massas de ar. Acreditando que a proximidade das estações meteorológicas minimizaram a influência das massas de ar sob os resultados, credita-se ao bioma e amaritimidade a maior parcela de contribuição sobre a diferença nas entropias observadas nas diferentes regiões. Ou seja, estes resultados são um forte indício da influência do bioma sobre a entropia de variáveis climáticas, onde destaca-se neste bioma uma maior regularidade temporal das médias diárias da temperatura e da umidade relativa do ar.

\section{Conclusão}

O estudo realizado propôs estudar a evolução temporal da entropia em variáveis climáticas no município de Poço Redondo-SE, com o uso da técnica Sample Entropy. Os valores de entropia estimados são considerados medianos para a temperatura média diária do ar e umidade relativa média diária do ar, e alto para a velocidade média diária do vento, onde as entropias estimadas para a velocidade do vento foram predominantemente maiores do que as estimativas para as outras variáveis. Apenas no ano de 2009 a entropia estimada para a umidade relativa média diária do ar foi menor que a mesma estatística estimada para temperatura média diária do ar, nos demais anos a estatística estimada para a umidade relativa do ar foi superior as estimativas para a mesma estatística estimada para temperatura do ar. Não foram observadas tendências nas séries entrópicas, onde todas foram caracterizadas como estacionárias para a janela estudada. Em comparação com o observado por Santos et al. (2015), no município de Estância, foi possível observar a influência da maritimidade e do bioma sob a 
entropia calculada para as três variáveis, onde notou-se uma maior regularidade das séries diárias de temperatura e umidade relativa do ar no bioma Caatinga, e uma maior entropia da velocidade do vento no bioma Mata Atlântica.

\section{REFERENCIAS}

Balzter H, Tate N, Kaduk J, Harper D, Page S, et al. 2015. Multi-Scale Entropy Analysis as a Method for TimeSeries Analysis of Climate Data. Climate, 3(1): 227-240.

Benicio RB de, Stošić T, Figueirêdo PH de, Stošić BD. 2013. Multifractal behavior of wild-land and forest fire time series in Brazil. Physica A: Statistical Mechanics and Its Applications, 392(24): 6367-6374.

Cruz MAS. 2009. Regionalização de precipitações médias e prováveis mensais e anuais no estado de Sergipe. Aracaju-SE: EMBRAPA.

Figueirêdo BCL de, Moreira GR, Stosic B, Stosic T. 2014. Multifractal analysis of hourly wind speed records in Petrolina, Northeast Brazil. Revista Brasileira de Biometria, 32(4): 599-608.

Filho AC, Maluf JRT, Matzenauer R. 2008. Coordenadas geográficas na estimativa das temperaturas máxima e média decendiais do ar no Estado do Rio Grande do Sul. Ciência Rural, 38(9): 2448-2456.

IBGE. 2002. Atlas Geográfico Escolar. Rio de Janeiro - RJ: Instituto Brasileiro de Geografia e Estatística.

IBGE. 2014. IBGE -Downloads. Instituto Brasileiro de Geografia e Estatística. http:/ / downloads.ibge.gov. br/downloads_geociencias.htm.

Ismael-Filho A, Borges P de F, Araújo L de S, Pereira RA, Lima EM de, et al. 2015. Influência das variáveis climáticas sobre a evapotranspiração. Gaia Scientia, 9(1): 62-66.

Jongejans E, Telenius A. 2001. Field experiments on seed dispersal by wind in ten umbelliferous species (Apiaceae). Plant Ecology, 152(1): 67-78.

Jönsson P. 1992. Wind erosion on sugar beet fields in Scania, southern Sweden. Agricultural and Forest Meteorology, 62(3-4): 141-157.

Kossobokov V, Mouël J-L Le, Allègre C. 2012. Spatial and Temporal Variations of Climate in Europe. Atmospheric and Climate Sciences, 2(4): 568-581.

Li T, Zheng X, Dai Y, Yang C, Chen Z, et al. 2014. Mapping near-surface air temperature, pressure, relative humidity and wind speed over Mainland China with high spatiotemporal resolution. Advances in Atmospheric Sciences, 31(5): 1127-1135.

Li-Hao G, Zun-Tao F. 2013. Multi-fractal Behaviors of Relative Humidity over China. Atmospheric and Oceanic Science Letters, 6(2): 74-78.

Mendonça F, Danni-Oliveira IM. 2007. Climatologia: noções básicas e climas do Brasil. São Paulo: Oficina 
de Textos.

Mihailovic DT, Nikolic-Djoric E, Dreskovic N, Mimic G. 2014. Complexity analysis of the turbulent environmental fluid flow time series. Physica A: Statistical Mechanics and Its Applications, 26: 96-104.

Pereira AR, Costa A de S, Oliveira VG de, Borges P de F, Filho AI. 2015. Análise do comportamento das médias anuais da precipitaçãoa pluvial e temperatura da cidade de Areia, Paraíba. Gaia Scientia, 9: 241-260.

Pimenta F, Kempton W, Garvine R. 2008. Combining meteorological stations and satellite data to evaluate the offshore wind power resource of Southeastern Brazil. Renewable Energy, 33(11): 2375-2387.

Pincus SM, Viscarello RR. 1992. Approximate entropy: a regularity measure for fetal heart rate analysis. Obstetrics and Gynecology, 79(2): 249-55.

Quadro MFL de, Dias MAF da S, Herdies DL, Gonçalves LGG de. 2012. Análise climatológica da precipitação e do transporte de umidade na região da ZCAS através da nova geração de reanálises. Revista Brasileira de Meteorologia, 27(2): 152-162.

Richman JS, Moorman JR. 2000. Physiological time-series analysis using approximate entropy and sample entropy. American Journal of Physiology. Heart and Circulatory Physiology, 278(6): H2039-49.

Santos ALS, Araújo L de S, Silva JRS, Farias P de B. 2015. Evolução temporal da entropia de variáveis climáticas em Estância-SE. Gaia Scientia, 9(1): 146-150.

Shuangcheng L, Qiaofu Z, Shaohong W, Erfu D. 2006. Measurement of climate complexity using sample entropy. International Journal of Climatology, 26(15): 2131-2139.

Silva JRS. 2014. Avaliação de autocorrelações e complexidade de séries temporais climáticas no Brasil. Recife - PE: Tese (Doutorado em Biometria e Estatística Aplicada) - Universidade Federal Rural de Pernambuco.

Slate JE, Johnson TC, Moore TC. 2013. Impact of pre-Columbian agriculture, climate change, and tectonic activity inferred from a 5,700-year paleolimnological record from Lake Nicaragua. Journal of Paleolimnology, 50(1): 139-149.

Tang X, Tian X, Yang Z, Zhang T. 2004. Complexity measurements of electroencephalograph recordings using sample entropy algorithm in patients with temporal lobe epilepsy. Acta Biophysica Sinica, 20: 382-392.

Torres FTP, Machado PJO. 2008. Introdução à Climatologia. Santo André - SP: Geographica.

Varejão-Silva MA. 2006. Meteorologia e Climatologia (2nd ed.). Recife - PE: Versão Digital.

Zhao ZQ, Li SC, Gao JB, Wang YL. 2011. Identifying spatial patterns and dynamics of climate change using recurrence quantification analysis: a case study of qinghai-tibet plateau. International Journal of Bifurcation and Chaos, 21(4): 1127-1139. 